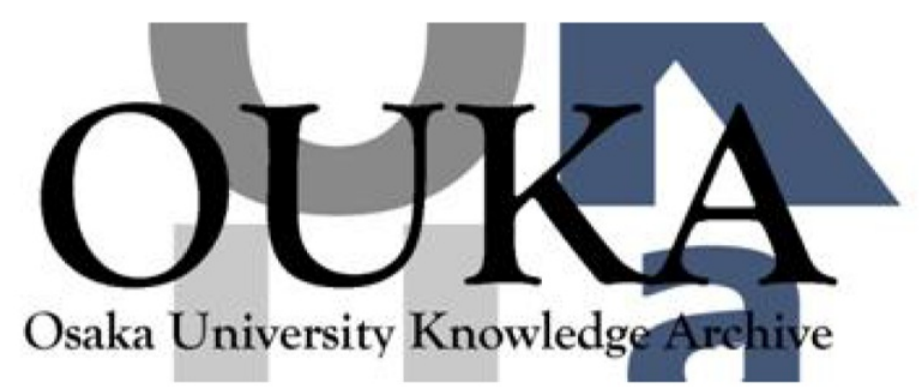

\begin{tabular}{|c|l|}
\hline Title & Collectivity of neutron-rich Ti isotopes \\
\hline Author(s) & Suzuki, H.; Aoi, N.; Takeshita, E. et al. \\
\hline Citation & $\begin{array}{l}\text { Physical Review C - Nuclear Physics. 88(2) } \\
\text { p. 24326-p. 24326 }\end{array}$ \\
\hline Issue Date & $2013-08-30$ \\
\hline oaire:version & VoR \\
\hline URL & https://hdl. handle. net/11094/51211 \\
\hline rights & o2013 American Physical Society \\
\hline Note & \\
\hline
\end{tabular}

Osaka University Knowledge Archive : OUKA

https://ir. Library. osaka-u. ac. jp/

Osaka University 


\title{
Collectivity of neutron-rich Ti isotopes
}

H. Suzuki, ${ }^{1,2}$ N. Aoi, ${ }^{1,3}$ E. Takeshita, ${ }^{1,4}$ S. Takeuchi, ${ }^{1}$ S. Ota, ${ }^{5}$ H. Baba, ${ }^{1}$ S. Bishop, ${ }^{1}$ T. Fukui, ${ }^{5}$ Y. Hashimoto, ${ }^{6}$ E. Ideguchi, ${ }^{7}$ K. Ieki, ${ }^{4}$ N. Imai,${ }^{8}$ M. Ishihara, ${ }^{1}$ H. Iwasaki, ${ }^{2,9,10}$ S. Kanno, ${ }^{4}$ Y. Kondo, ${ }^{6}$ T. Kubo, ${ }^{1}$ K. Kurita, ${ }^{4}$ K. Kusaka, ${ }^{1}$ T. Minemura, ${ }^{8}$ T. Motobayashi, ${ }^{1}$ T. Nakabayashi, ${ }^{6}$ T. Nakamura, ${ }^{6}$ T. Nakao, ${ }^{2}$ M. Niikura, ${ }^{2,7}$ T. Okumura, ${ }^{6}$ T. K. Ohnishi, ${ }^{2}$ H. J. Ong, ${ }^{2,3}$ H. Sakurai, ${ }^{2}$ S. Shimoura, ${ }^{7}$ R. Sugo, ${ }^{4}$ D. Suzuki, ${ }^{2,11}$ M. K. Suzuki, ${ }^{2}$ M. Tamaki, ${ }^{7}$ K. Tanaka, ${ }^{1}$ Y. Togano, ${ }^{4,6}$ and K. Yamada ${ }^{1}$ ${ }^{1}$ RIKEN Nishina Center, 2-1 Hirosawa, Wako, Saitama 351-0198, Japan

${ }^{2}$ Department of Physics, University of Tokyo, 7-3-1 Hongo, Bunkyo, Tokyo 113-0033, Japan

${ }^{3}$ RCNP, Osaka University, 10-1 Mihogaoka, Ibaraki, Osaka 567-0047, Japan

${ }^{4}$ Department of Physics, Rikkyo University, 3-34-1 Nishi-Ikebukuro, Toshima, Tokyo 172-8501, Japan

${ }^{5}$ Department of Physics, Kyoto University, Kitashirakawa, Kyoto 606-8502, Japan

${ }^{6}$ Department of Physics, Tokyo Institute of Technology, 2-12-1 Ookayama, Meguro, Tokyo 152-8551, Japan

${ }^{7}$ Center for Nuclear Study, University of Tokyo, RIKEN campus, 2-1 Hirosawa, Wako, Saitama 351-0298, Japan

${ }^{8}$ Institute of Particle and Nuclear Study, KEK, 1-1 Oho, Tsukuba 305-0801, Japan

${ }^{9}$ National Superconducting Cyclotron Laboratory, Michigan State University, East Lansing, Michigan 48824, USA

${ }^{10}$ Department of Physics and Astronomy, Michigan State University, East Lansing, Michigan 48824, USA

${ }^{11}$ Institut de Physique Nucléaire, IN2P3-CNRS, Université de Paris-Sud, F-91406 Orsay Cedex, France

(Received 13 April 2012; revised manuscript received 22 July 2013; published 30 August 2013)

\begin{abstract}
The structure of the neutron-rich nucleus ${ }^{58} \mathrm{Ti}$ was investigated via proton inelastic scattering in inverse kinematics at a mean energy of $42.0 \mathrm{MeV} /$ nucleon. By measuring the deexcitation $\gamma$ rays, three transitions with the energies of $1046(11) \mathrm{keV}, 1376(18) \mathrm{keV}$, and $1835(27) \mathrm{keV}$ were identified. The angle-integrated cross section for the 1046-keV excitation, which corresponds to the decay from the first $2^{+}$state, was determined to be 13(7) mb. The deformation length $\delta_{p, p^{\prime}}$ was extracted from the cross section to be $0.83_{-0.30}^{+0.22} \mathrm{fm}$. The energy of the first $2^{+}$state and the $\delta_{p, p^{\prime}}$ value are comparable to the ones of ${ }^{56} \mathrm{Ti}$, which indicates that the collectivity of the $\mathrm{Ti}$ isotopes does not increase significantly with neutron number until $N=36$. This fact indicates that ${ }^{58} \mathrm{Ti}$ is outside of the region of the deformation known in the neutron-rich nuclei around $N=40$.
\end{abstract}

DOI: 10.1103/PhysRevC.88.024326

PACS number(s): 21.10.-k, 27.50.+e, 25.60.-t, 25.40.Ep

\section{INTRODUCTION}

The shell structure as the basis of our understanding of the atomic nucleus has recently been found to change drastically due to the rearrangement of the single particle levels as one moves away from the stability line. In unstable nuclei with very asymmetric proton and neutron numbers, various exotic properties have been revealed such as the anomalous deformation emerging in the very neutron-rich regions around $N=8[1-3]$ and 20 [4,5]. These neutron numbers, which are considered to be magic in the traditional nuclear theory, lose the magicity in the neutron-rich region, and the nuclei with these neutron numbers become accordingly deformed. In order to account for such anomalous deformation phenomena, several theoretical models have been proposed, although the mechanism is still unclear. The new region of deformation recently found in the neutron-rich $p f$-shell region around $N=40[6,7]$ may provide us a clue to clarify the mechanism.

$N=40$ is a magic number for a harmonic oscillator potential, but its energy gap is narrowed by the intruder $g_{9 / 2}$ orbital lowered by the spin-orbit interaction. Because of the fragile energy gap, the nuclei with $N=40$ exhibit a variety of characters depending on the proton number. For the $\mathrm{Ni}$ isotopes with a proton magic number $Z=28$, ${ }^{68} \mathrm{Ni}$ shows small collectivity reflecting the magic character of $N=40$ as indicated by its large value of the energy of the first $2^{+}$excited state $\left[E_{\mathrm{x}}\left(2_{1}^{+}\right)\right]$and small value of quadrupole transition probability $[B(\mathrm{E} 2)][8,9]$. However, in
Fe isotopes $(Z=26)$, the $E_{\mathrm{x}}\left(2_{1}^{+}\right)$values start decreasing at $N=38$, and this decrease continues monotonically beyond $N=40[10,11]$. This indicates that $N=40$ does not exhibit a magic character in $\mathrm{Fe}$ anymore [12,13]. In the $\mathrm{Cr}$ isotopes $(Z=24)$, the enhancement of the collectivity is seen already in the nuclei with $N=36$, where the $E_{\mathrm{x}}\left(2_{1}^{+}\right)$values drop much below the typical value of the open neutron-shell nuclei along the $\mathrm{Cr}$ isotopic chain [7,14]. The decrease continues until ${ }^{64} \mathrm{Cr}$, in which $E_{\mathrm{x}}\left(2_{1}^{+}\right)$value shows the smallest among the known $E_{\mathrm{x}}\left(2_{1}^{+}\right)$values in this region [15]. The enhancement of collectivity toward $N=40$ in the Cr isotopes was confirmed by the rapid increase of deformation lengths $(\delta)$ and the $E_{\mathrm{x}}\left(4_{1}^{+}\right) / E_{\mathrm{x}}\left(2_{1}^{+}\right)$ratios $\left(R_{4 / 2}\right)[14,16]$. A question arises as to whether the anomalous deformation region found in $\mathrm{Fe}$ and $\mathrm{Cr}$ isotopes further develops in more proton-deficient isotopes, in particular Ti.

Theoretically, a deformation region is predicted to appear around ${ }^{62} \mathrm{Ti}$ [6]. Indeed, the enhancement of collectivity has been suggested for the Ti isotopes in this region from their $\beta$-decay half-lives. Approaching $N=40$ from ${ }^{58} \mathrm{Ti}$ to ${ }^{60} \mathrm{Ti}$, the $\beta$-decay half-lives become gradually longer than the shell model calculation within the $p f$ shell, which is interpreted as being due to the admixture of the neutron $v g_{9 / 2}$ configuration [17]. Considering the deformation-driving nature of the $\nu g_{9 / 2}$ orbital, the increase of the $\nu g_{9 / 2}$ contribution would enhance the collectivity. To locate the boundary of the anomalous deformation region in the Ti isotopes, the study of the collectivity of $\mathrm{Ti}$ isotopes is of great importance. 
We have investigated the properties of ${ }^{58} \mathrm{Ti}$, through the low-lying states including the first $2^{+}$state, whose preliminary result was partially reported in Ref. [18]. We utilized the proton inelastic scattering in inverse kinematics to populate these states, which enables us to extract the $\delta$ value as well as the excitation energies. These two quantities [ $\delta$ and $\left.E_{\mathrm{x}}\left(2_{1}^{+}\right)\right]$are essential for the discussion of the quadrupole collectivity. This measurement was possible even with a low-intensity beam due to the high efficiency and high signal-to-noise ratio of the measurement achieved by the use of the $\gamma$-ray spectroscopy technique incorporating a thick liquid-hydrogen target. In this paper, we report on the $\delta$ value and two new $\gamma$ transitions as well as the $E_{\mathrm{x}}\left(2_{1}^{+}\right)$value.

\section{EXPERIMENT}

The experiment was performed at RI Beam Factory operated by the RIKEN Nishina Center and Center for Nuclear Study, University of Tokyo, using the same experimental arrangement described in Ref. [14]. A cocktail beam composed of several nuclear species including ${ }^{58} \mathrm{Ti}$ was produced by the fragmentation reaction of a ${ }^{70} \mathrm{Zn}$ primary beam with a typical intensity of $150 \mathrm{p} \mathrm{nA}$ impinging on a $96-\mathrm{mg} / \mathrm{cm}^{2}(0.52-\mathrm{mm})$ Be target at $63 \mathrm{MeV} /$ nucleon. The fragments produced were collected and analyzed by the RIKEN projectile-fragment separator (RIPS) [19]. The particle identification (PID) of the cocktail beam was made on an event-by-event basis. The average intensity of ${ }^{58} \mathrm{Ti}$ was 1.5 counts $/ \mathrm{s}$, while the total beam intensity was $1 \times 10^{3}$ counts $/ \mathrm{s}$.

A $72-\mathrm{mg} / \mathrm{cm}^{2}$ thick liquid-hydrogen produced by the "cryogenic proton and alpha target system" (CRYPTA) [20] was bombarded by the secondary beam inducing excitation of ${ }^{58} \mathrm{Ti}$ via proton inelastic scattering. The energy of ${ }^{58} \mathrm{Ti}$ at the center of the secondary target was $42.0 \mathrm{MeV} /$ nucleon.

The levels populated by the secondary reactions were identified by measuring the deexcitation $\gamma$ rays in coincidence with the reaction residues. The $\gamma$ rays were measured by DALI2 [21], which consisted of $160 \mathrm{NaI}(\mathrm{Tl})$ scintillators surrounding the secondary target. The $\gamma$-ray energies were corrected for the Doppler shift using the angle between the scattered particle and the triggered crystal. The spectral shape and efficiency of $\gamma$ rays were obtained by a Monte Carlo simulation using the GEANT code [22]. The energy resolution and full-energy peak efficiency for 1046-keV $\gamma$ rays emitted from a moving ${ }^{58} \mathrm{Ti}$ projectile were determined to be $8.5 \%$ (FWHM) and 18\%, respectively.

The scattered particles were identified using the "TOF mass analyzer for RI beam experiments" (TOMBEE) [23] by measuring their TOF, $\Delta E$, and total kinetic energy $(E)$. The atomic number $Z$ and the mass number $A$ were obtained from the TOF- $\Delta E$ and TOF- $E$ correlations with resolutions of $1.5 \%$ and $1.2 \%$ (FWHM), respectively. The good mass resolution of $1.2 \%$ enabled the unambiguous identification of the reaction residues in the medium-mass region around $A \sim 60$. The transmission of TOMBEE was evaluated by the ion optics code COSY INFINITY [24] to be about $90 \%$ at 0 degrees, decreasing with the scattering angle to $50 \%$ at 2 degrees. The total transmission integrated over the scattering angle is $88 \%$ for nonreacted events and $87 \%$ for inelastically scattered events.

\section{RESULTS}

The Doppler-shift corrected energy spectrum of $\gamma$ rays measured in coincidence with inelastically scattered ${ }^{58} \mathrm{Ti}$ is shown in Fig. 1(a). Three peaks at 1046(11) keV, 1376(18) keV, and $1835(27) \mathrm{keV}$ were clearly identified. The quoted errors include the statistical ones and uncertainties in the energy calibration. The energy of the $1046-\mathrm{keV}$ peak is consistent with our earlier report [1046(17) keV] [18], while the error is reduced. The 1046-keV peak was observed most strongly, and was thus assigned to the transition from a newly identified first $2^{+}$state to the $0^{+}$-ground state.

In the $\gamma$-ray spectrum gated on the $1046-\mathrm{keV}$ peak shown in Fig. 1(c), the $\gamma$ line at $1835 \mathrm{keV}$ is observed. This transition was therefore assigned to be the one from a level at $2881(33) \mathrm{keV}$ to the first $2^{+}$state as illustrated in Fig. 2. In the region around $1376 \mathrm{keV}$, two events are observed, which correspond most probably to the full-energy peak of the 1376-keV $\gamma$ rays observed in coincidence with the $2_{1}^{+} \rightarrow 0_{\text {g.s. }}^{+}$transition. It is less likely that these events are due to coincidence with the Compton scattering of the $1835-\mathrm{keV} \gamma$ rays, since the probability of observing more than two events due to such a process is estimated to be $18 \%$.

The angle-integrated cross section for the direct excitation to the first $2^{+}$state in ${ }^{58} \mathrm{Ti}$ was obtained from the peak yield of

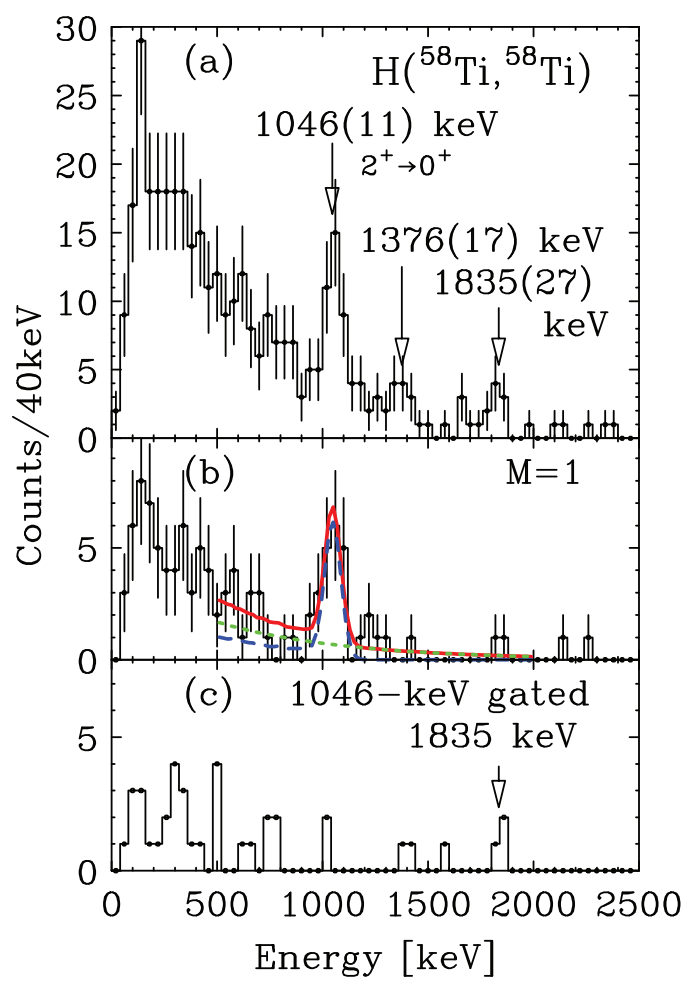

FIG. 1. (Color online) (a) Doppler-shift corrected $\gamma$-ray energy spectrum for the proton inelastic scattering on ${ }^{58} \mathrm{Ti}$. (b) The histogram is of the $M=1$ events. (c) $\gamma$-ray spectrum gated on the $1046-\mathrm{keV} \gamma$ line. 


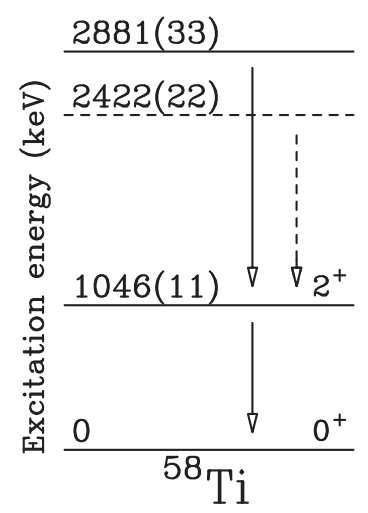

FIG. 2. Level scheme of ${ }^{58} \mathrm{Ti}$ proposed in this work.

the $2_{1}^{+} \rightarrow 0_{\text {g.s. }}^{+}$transition with the $\gamma$-ray multiplicity equal to 1 (denoted $\stackrel{M}{M}=1$ hereafter) as shown in Fig. 1(b). The peak yield was extracted by fitting the spectrum with the sum (solid curve) of the response function of DALI2 for the $2_{1}^{+} \rightarrow 0_{\text {g.s. }}^{+}$ transition (dashed curve) and an exponential background (dotted curve). The condition of $M=1$ was intended to eliminate the $2_{1}^{+} \rightarrow 0_{\text {g.s. }}^{+}$transitions which is accompanied by cascade emission of $\gamma$ rays from higher excited states. Because of the high total detection efficiency of $\gamma$ rays $(\sim 70 \%)$, most of the cascade events were eliminated by this condition. The contamination by the cascade events in the $M=1$ spectrum was estimated to be $22 \%$ by the Monte Carlo simulation and was corrected for. It was found that the contributions of the direct excitation to the first $2^{+}$state amount to $34(16) \%$ of the observed $2_{1}^{+} \rightarrow 0_{\text {g.s. }}^{+}$transition.

The first $2^{+}$excitation cross section was determined to be 13(7) $\mathrm{mb}$, after correcting for the transmission and the $\gamma$-ray detection efficiency. The quoted error includes the statistical one $(31 \%)$, and the uncertainties in the target thickness $(5 \%)$, the $\gamma$-ray detection efficiency $(4 \%)$, and the cascade subtraction (16\%).

In order to extract the deformation length $\delta_{p, p^{\prime}}$, the calculations based on the distorted-wave theory were performed using the ECIS97 code [25]. The collective vibrational model was adopted with four sets of optical potential parameters [26-29]. The average for $\delta_{p, p^{\prime}}$ is $0.83_{-0.30}^{+0.22} \mathrm{fm}$. The error includes the uncertainty of the experimental cross section and the one due to the choice of the potential parameters $(4 \%)$. The corresponding deformation parameter $\beta_{2}\left(=\delta_{p, p^{\prime}} / R\right)$ is $0.18_{-0.06}^{+0.05}$ using the nuclear radii given by $R=r_{0} A^{1 / 3}\left(r_{0}=1.2 \mathrm{fm}\right)$.

\section{DISCUSSION}

The $E_{\mathrm{x}}\left(2_{1}^{+}\right)$value of ${ }^{58} \mathrm{Ti}$ obtained in this work is compared with those of the lighter even-even Ti isotopes in Fig. 3(a) (circles). The $E_{\mathrm{x}}\left(2_{1}^{+}\right)$value of ${ }^{58} \mathrm{Ti}$ is slightly smaller than that of ${ }^{56} \mathrm{Ti}$, but is not significantly smaller than those of the lighter open-neutron-shell $\mathrm{Ti}$ isotopes. This indicates that the collectivity is not particularly enhanced in ${ }^{58} \mathrm{Ti}$ with $N=36$. Therefore, ${ }^{58} \mathrm{Ti}$ is outside of the region of large collectivity known in the $\mathrm{Fe}$ and $\mathrm{Cr}$ isotopes close to $N=40$. The region of large collectivity emerges in the Fe isotopes from

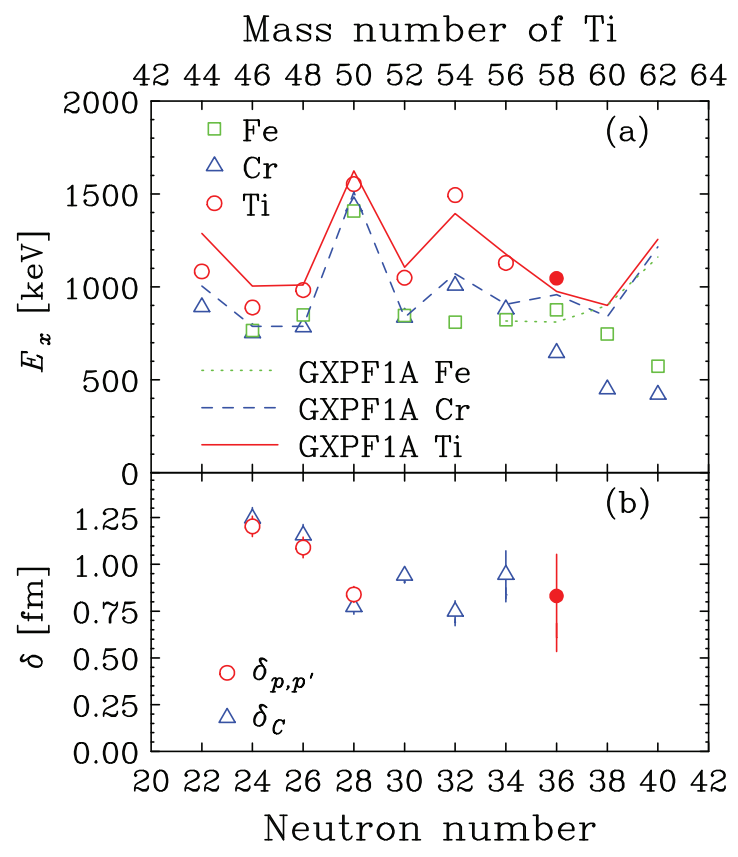

FIG. 3. (Color online) (a) Plot of the excitation energies of the first $2^{+}$states as a function of the neutron number for the $\mathrm{Ti}$ (circles), $\mathrm{Cr}$ (triangles), and $\mathrm{Fe}$ (squares) isotopes. The filled circle is the result from the present work. Solid, dashed, and dotted lines are the results of the shell model calculation within the $p f$ shell using the GXPF1A interaction for $\mathrm{Ti}, \mathrm{Cr}$, and $\mathrm{Fe}$ isotopes, respectively [30-32]. (b) Plot of $\delta_{p, p^{\prime}}$ for the Ti isotopes as a function of the neutron number. The filled circle is the result from the present work; open circles are those of the stable Ti isotopes taken from Ref. [29]. Triangles show $\delta_{C}$ estimated from the $B(E 2)$ values.

$N=38$ [Fig. 3(a) (squares)], and then develops further in the $\mathrm{Cr}$ isotopes [Fig. 3(a) (triangles)] in a wider region starting from $N=36$. Our finding shows that the region of large collectivity is not extended to the Ti isotopes at $N=36$ as in the $\mathrm{Cr}$ isotopes. The slight decrease of the $E_{\mathrm{x}}\left(2_{1}^{+}\right)$value in ${ }^{58} \mathrm{Ti}$ could be a precursor of the drop in more neutron-rich isotopes due to a possible enhancement of collectivity.

The experimental values were compared with the results of the shell model calculation within the $p f$ shell using the GXPF1A interaction [30-32] in Fig. 3(a). In the Ti isotopes, the $E_{\mathrm{x}}\left(2_{1}^{+}\right)$values obtained with the calculation show an overall agreement with the experimental values including the one for ${ }^{58} \mathrm{Ti}$. For the neighboring isotopes, the calculation deviates from the experimental $E_{\mathrm{x}}\left(2_{1}^{+}\right)$values beyond $N=38$ in the $\mathrm{Fe}$ isotopes and beyond 36 in the $\mathrm{Cr}$ isotopes. In this region, the experimental values start decreasing, while the theoretical values remain almost constant and then increase at $N=40$. The deviation in $\mathrm{Fe}$ and $\mathrm{Cr}$ isotopes indicates that the $p f$-shell model space is not sufficient to describe these nuclei, and that the contribution of the $g_{9 / 2}$-shell configuration has to be considered $[14,33]$. The good agreement between the experimental and theoretical $E_{\mathrm{x}}\left(2_{1}^{+}\right)$values in ${ }^{58} \mathrm{Ti}$ demonstrates that the contribution of the $g_{9 / 2}$ shell is not significant in this nucleus.

The $\delta_{p, p^{\prime}}$ value of ${ }^{58} \mathrm{Ti}$ is plotted in Fig. 3(b) (filled circle) along with the ones for the stable $\mathrm{Ti}$ isotopes, ${ }^{46,48,50} \mathrm{Ti}$ 
(open circles). The triangles show the Coulomb deformation lengths $\left(\delta_{C}\right)$ deduced from $B(E 2)$. Both values are measures of the nuclear deformation; $\delta_{p, p^{\prime}}$ reflects proton and neutron distribution, while $\delta_{C}$ reflects proton distribution. We assume that $\delta_{p, p^{\prime}}$ and $\delta_{C}$ are similar to each other, and use the both values to trace the evolution of the collectivity. The $\delta$ value of ${ }^{58} \mathrm{Ti}$ is about the same as that of ${ }^{56} \mathrm{Ti}$ within the experimental error. This agreement is consistent with the conclusion drawn from the $E_{\mathrm{x}}\left(2_{1}^{+}\right)$values that the collectivity does not increase significantly at ${ }^{58} \mathrm{Ti}$.

The behavior of the collectivity in the nuclei in this region could be qualitatively understood as the result of competition between the following two effects. One is that the valence protons partially fill the $\pi f_{7 / 2}$ shell, which favors deformation [34]. The other is narrowing of the energy spacing at $N=40$ between $v f_{5 / 2}$ and $v g_{9 / 2}$ by the strong protonneutron interaction [7,30-32]. The monopole component of the proton-neutron interaction is attractive between $\pi f_{7 / 2}$ and $v f_{5 / 2}$, and repulsive between $\pi f_{7 / 2}$ and $v g_{9 / 2}$. When protons are removed from the $\pi f_{7 / 2}$ orbital, $v f_{5 / 2}$ is shifted upward and $v g_{9 / 2}$ is shifted downward, resulting in the narrowing of the energy spacing between $v f_{5 / 2}$ and $v g_{9 / 2}$. The latter effect should increase monotonically as number of protons in $\pi f_{7 / 2}$ decreases from $\mathrm{Ni}$ down to $\mathrm{Ca}$. On the other hand, the former effect should increase in the beginning, but would start decreasing beyond the $\mathrm{Cr}$ isotopes in which half of the $\pi f_{7 / 2}$ shell is filled. The experimental $E_{\mathrm{x}}\left(2^{+}\right)$values along the $N=$ 36 isotonic chain shows that the collectivity increases from ${ }^{64} \mathrm{Ni}$ to ${ }^{60} \mathrm{Cr}$, and then decreases in ${ }^{58} \mathrm{Ti}$. This behavior suggests that the former effect is stronger than the latter in the $N=36$ isotones. Approaching $N=40$, the balance between these two effects may change. The mechanism of the deformation in the neutron-rich $p f$-shell nuclei should be revealed by studying the evolution of the collectivity in a wider region of nuclei.

\section{SUMMARY}

In summary, we have investigated the structure of the neutron-rich nucleus ${ }^{58} \mathrm{Ti}$ via proton inelastic scattering in inverse kinematics at a mean energy of $42.0 \mathrm{MeV} /$ nucleon. By measuring the deexcitation $\gamma$ rays, three transitions with energies of 1046(11) $\mathrm{keV}, 1376(18) \mathrm{keV}$, and 1835(27) $\mathrm{keV}$ were identified. The energy of the $1046-\mathrm{keV}$ peak, which corresponds to the first $2^{+}$state, is consistent with our earlier report [18]. The 1376-keV and $1835-\mathrm{keV}$ transitions were assigned as the ones from the levels at 2422(22) $\mathrm{keV}$ and $2881(33) \mathrm{keV}$ to the first $2^{+}$state, respectively, where the former assignment is a tentative one. The angle-integrated cross section for the excitation to the first $2^{+}$state was determined to be 13(7) mb. The deformation length $\delta_{p, p^{\prime}}$ was extracted from the cross section to be $0.83_{-0.30}^{+0.22} \mathrm{fm}$. The $E_{\mathrm{x}}\left(2_{1}^{+}\right)$ value and the deformation length of ${ }^{58} \mathrm{Ti}$ are comparable to the ones of ${ }^{56} \mathrm{Ti}$. These values indicate that the collectivity of the Ti isotopes does not change significantly with neutron number until $N=36$, which is in contrast to the $\mathrm{Cr}$ isotopes whose collectivity starts increasing at $N=36$. This fact indicates that ${ }^{58} \mathrm{Ti}$ is outside of the region of the deformation known in the neutron-rich nuclei around $N=40$. From the comparison to a shell model calculation, ${ }^{58} \mathrm{Ti}$ is found to be well described within the $p f$-shell model space.

\section{ACKNOWLEDGMENTS}

We thank the RIKEN Ring Cyclotron staff members for providing the stable primary beam throughout the experiment. We would like to thank Dr. M. Honma for fruitful discussions. One of the authors (H.S.) would like to acknowledge support from a research grant of the Japan Society for the Promotion of Science.
[1] A. Navin et al., Phys. Rev. Lett. 85, 266 (2000).

[2] S. Shimoura et al., Phys. Lett. B 654, 87 (2007).

[3] H. Iwasaki et al., Phys. Lett. B 481, 7 (2000).

[4] D. Guillemaud-Mueller, C. Detraz, M. Langevin, F. Naulin, M. De Saint-Simon, C. Thibault, F. Touchard, and M. Epherre, Nucl. Phys. A 426, 37 (1984).

[5] T. Motobayashi et al., Phys. Lett. B 346, 9 (1995).

[6] B. A. Brown, Prog. Part. Nucl. Phys. 47, 517 (2001).

[7] O. Sorlin et al., Eur. Phys. J. A 16, 55 (2003).

[8] O. Sorlin et al., Phys. Rev. Lett. 88, 092501 (2002).

[9] O. Perru et al., Phys. Rev. Lett. 96, 232501 (2006).

[10] A. N. Deacon et al., Phys. Rev. C 76, 054303 (2007).

[11] P. Adrich et al., Phys. Rev. C 77, 054306 (2008).

[12] J. Ljungvall et al., Phys. Rev. C 81, 061301 (2010).

[13] W. Rother et al., Phys. Rev. Lett. 106, 022502 (2011).

[14] N. Aoi et al., Phys. Rev. Lett. 102, 012502 (2009).

[15] A. Gade et al., Phys. Rev. C 81, 051304 (2010).

[16] T. Baugher et al., Phys. Rev. C 86, 011305 (2012).

[17] L. Gaudefroy et al., Eur. Phys. J. A 23, 41 (2005).

[18] N. Aoi et al., Nucl. Phys. A 805, 400 (2008).
[19] T. Kubo, M. Ishihara, N. Inabe, H. Kumagai, I. Tanihata, K. Yoshida, T. Nakamura, H. Okuno, S. Shimoura, and K. Asahi, Nucl. Instrum. Methods Phys. Res. B 70, 309 (1992).

[20] H. Ryuto, M. Kunibu, T. Minemura, T. Motobayashi, K. Sagara, S. Shimoura, M. Tamaki, Y. Yanagisawa, and Y. Yano, Nucl. Instrum. Methods Phys. Res. A 555, 1 (2005).

[21] S. Takeuchi, T. Motobayashi, H. Murakami, K. Demichi, and H. Hasegawa, RIKEN Accel. Prog. Rep. 36, 148 (2003).

[22] GEANT3: Detector Description and Simulation Tool, CERN Program Library.

[23] N. Aoi et al., RIKEN Accel. Prog. Rep. 38, 176 (2005).

[24] K. Makino and M. Berz, Nucl. Instrum. Methods Phys. Res. A 558, 346 (2006).

[25] J. Raynal, coupled channel code ECIS97.

[26] R. L. Varner, W. J. Thompson, T. L. McAbee, E. J. Ludwig, and T. B. Clegg, Phys. Rep. 201, 57 (1991).

[27] A. J. Koning and J. P. Delaroche, Nucl. Phys. A 713, 231 (2003).

[28] F. D. Becchetti, Jr. and G. W. Greenlees, Phys. Rev. 182, 1190 (1969). 
[29] E. Fabrici, S. Micheletti, M. Pignanelli, F. G. Resmini, R. De Leo, G. D'Erasmo, and A. Pantaleo, Phys. Rev. C 21, 844 (1980).

[30] T. Otsuka, T. Matsuo, and D. Abe, Phys. Rev. Lett. 97, 162501 (2006).

[31] M. Honma, T. Otsuka, B. A. Brown, and T. Mizusaki, Phys. Rev. C 69, 034335 (2004).
[32] M. Honma, T. Otsuka, B. A. Brown, and T. Mizusaki, Eur. Phys. J. A 25, 499 (2005).

[33] S. M. Lenzi, F. Nowacki, A. Poves, and K. Sieja, Phys. Rev. C 82, 054301 (2010).

[34] H. Oba and M. Matsuo, Prog. Theor. Phys. (Kyoto) 120, 143 (2008). 